\title{
DISCRETE VARIABLE STRUCTURE SYSTEM WITH PSEUDO-SLIDING MODE
}

\author{
R. B. POTTS and X. YU \\ (Received 11 August 1989; revised 4 May 1990)

\begin{abstract}
Variable structure systems with sliding modes have been widely discussed and used in many different fields of applications. The precise behaviour at a switching surface is complicated because there the system is non-analytic. The damped simple harmonic oscillator with a nonlinear variable structure is discretised and analysed in detail, revealing the occurrence and structure of pseudo-sliding modes which give insight to the corresponding sliding modes for the continuous system. Necessary and sufficient conditions are obtained and the analysis illustrated with graphs from numerical solutions.
\end{abstract}

\section{Introduction}

Variable structure systems with sliding modes have been widely discussed [5] and used in many different fields of applications [6]. Essentially the systems are modelled mathematically by differential equations with discontinuous righthand sides [2]. The precise behaviour at a switching surface is complicated because there the system is non-analytic. Solutions are sometimes presented graphically with an unconvincing sketched-in wiggly line down the switching surface to represent the sliding mode. It is known [3] that the necessary and sufficient condition for the existence of a sliding mode for a continuous linear system is a necessary but not sufficient condition for the existence of a pseudo-sliding mode for its sampled linear system.

It is the first purpose of the present paper to illustrate the occurrence and structure of a sliding mode by considering a discrete variable structure system based on the damped harmonic oscillator. The discretisation is taken in such

\footnotetext{
${ }^{1}$ Applied Mathematics Department, The University of Adelaide, South Australia 5001.

(C) Copyright Australian Mathematical Society 1991, Serial-fee code 0334-2700/91
} 
a way that the discrete points in the phase plane lie exactly on the trajectories for the continuous system [4], except for points on the switching lines, because on these the continuous solution does not exist; then the discretisation is represented by an approximation which allows the switching lines to be crossed and recrossed to give a possible chattering pseudo-sliding mode. It is the second purpose of this paper to explore the relation between the system parameters and the sampling period. Necessary and sufficient conditions for the occurrence of a pseudo-sliding mode for the discrete system are derived and shown to give the correct results for the limiting case of the continuous system. The theory is illustrated by numerical results which indicate the somewhat bizarre possibilities that can arise.

\section{Damped harmonic oscillator: continuous system}

Consider the variable structure system [5] given in state space form as the ordinary differential equations

$$
\begin{aligned}
& \dot{x}_{1}=x_{2} \\
& \dot{x}_{2}= \begin{cases}-a x_{1}-f x_{2}, & \text { if } x_{1}\left(c x_{1}+x_{2}\right) \geq 0 \\
+a x_{1}-f x_{2}, & \text { if } x_{1}\left(c x_{1}+x_{2}\right)<0 .\end{cases}
\end{aligned}
$$

This system corresponds to a damped harmonic oscillator with $x_{1}$ representing displacement $y(t)$ say, with

$$
\ddot{y}(t)+f \dot{y}(t) \pm a y(t)=0 .
$$

In matrix form this system can be written

$$
\dot{\mathbf{x}}=\mathbf{A}^{ \pm} \mathbf{x}
$$

where

$$
\mathbf{A}^{ \pm}=\left[\begin{array}{cc}
0 & 1 \\
\mp a & -f
\end{array}\right]
$$

The variable structure implied in (2) corresponds to a switching line

$$
s=s(\mathbf{x})=c x_{1}+x_{2}=0,
$$

so that the top sign in (5) is used for regions I and III in Figure 1 and the bottom sign for regions II and IV.

The formal solution of the system is

$$
\mathbf{x}(t)=\exp \left(\mathbf{A}^{ \pm}\left(t-t_{0}\right)\right) \mathbf{x}\left(t_{0}\right)
$$

where

$$
\exp \left(\mathbf{A}^{ \pm} t\right)=\exp (-\alpha t)\left[\begin{array}{cc}
\cos (\beta t)+\alpha \beta^{-1} \sin (\beta t) & \beta^{-1} \sin (\beta t) \\
(\mp a) \beta^{-1} \sin (\beta t) & \cos (\beta t)-\alpha \beta^{-1} \sin (\beta t)
\end{array}\right],
$$




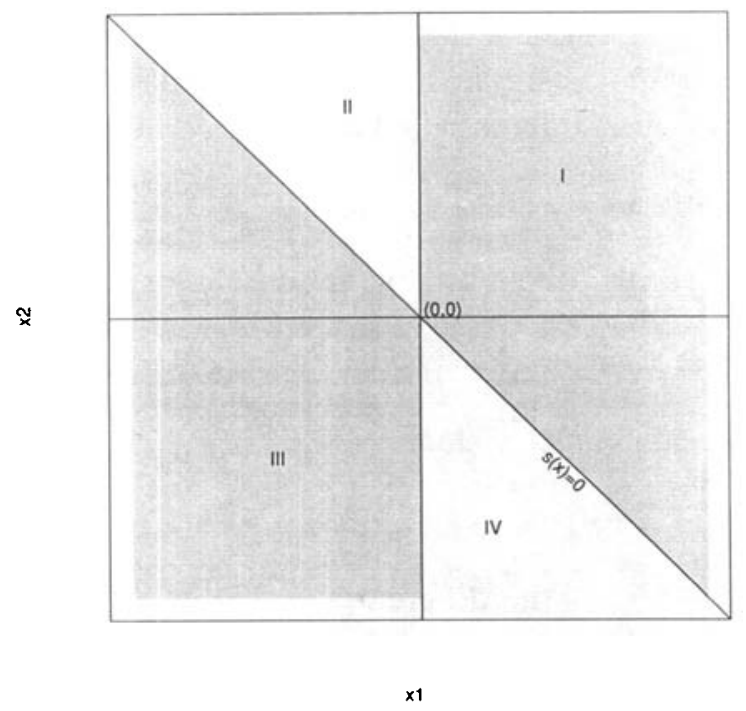

FIgURE 1. The $x_{1}, x_{2}$ phase plane is divided into regions corresponding to the sign of $x_{1} s(x)$ where $s(x)=x_{1}+x_{2}=0$ is the switching line. For the shaded regions I and III the top sign in (5) has to be taken; for II and IV the bottom sign.

with

$$
\begin{aligned}
& \alpha=f / 2, \\
& \beta=(1 / 2) \sqrt{ \pm 4 a-f^{2}} .
\end{aligned}
$$

The constant $\beta$ is real or pure imaginary.

It is well-known [1] that the necessary and sufficient conditions for $s(\mathbf{x})=0$ to be a sliding mode, characterised by $\dot{x}_{1}=-c x_{1}$, are

$$
\lim _{s \rightarrow+0} \dot{s} \leq 0, \quad \lim _{s \rightarrow-0} \dot{s} \geq 0 .
$$

Since

$$
\lim _{s \rightarrow+0} \dot{s}=\lim _{s \rightarrow-0} \dot{s}=x_{1}\left(c f-c^{2} \mp a\right),
$$

there exists a stable sliding mode for $a>0$ and $c>0$ provided that

$$
c^{2}-a \leq c f \leq c^{2}+a
$$

\section{Damped harmonic oscillator: discrete system}

We now consider a discretisation of the system by letting $\mathbf{x}(k)$ denote $\mathbf{x}$ evaluated at time $k h$, where $h$ is a discrete constant time interval. The 
system, apart from the variable structure, is a linear system. So ignoring for the moment the switching lines, the linear differential equation (4) can be discretised by the linear differential equation

$$
\mathbf{x}(k+1)=\exp \left(\mathbf{A}^{ \pm} h\right) \mathbf{x}(k), \quad k=0,1, \ldots
$$

In effect this replaces the differential equation (4) by the difference equation

$$
\mathbf{A}^{ \pm}\left(\exp \left(\mathbf{A}^{ \pm} h\right)-\mathbf{I}\right)^{-1}[\mathbf{x}(k+1)-\mathbf{x}(k)]=\mathbf{A}^{ \pm} \mathbf{x}(k),
$$

rather than the usual simpler system

$$
h^{-1}[\mathbf{x}(k+1)-\mathbf{x}(k)]=\mathbf{A}^{ \pm} \mathbf{x}(k) .
$$

The discretisation (15) of the differential equation (4) is the 'best', in the sense that, from any given initial point in the phase plane, the solution (14) of (15) gives discrete points exactly on the corresponding solution curve (7) of (4). This result is true regardless of the magnitude of $h$ [4].

We now take into account the nonlinear variable structure represented by (2). The discrete variable structure which decides which of the matrices $\mathbf{A}^{ \pm}$ to use is taken to be:

$$
\begin{aligned}
\text { use top sign } & \text { if } x_{1}(k)\left(c x_{1}(k)+x_{2}(k)\right) \geq 0 \\
\text { use bottom sign } & \text { if } x_{1}(k)\left(c x_{1}(k)+x_{2}(k)\right)<0 .
\end{aligned}
$$

Whereas the trajectory for the continuous system stops if it reaches the switching line $s(\mathbf{x})=0$, the discretisation allows the trajectory to cross the line $s(k)=c x_{1}(k)+x_{2}(k)=0$. If it does cross, then the matrix is changed to that with the opposite designated sign. The particular details of the crossing of the line and the subsequent discrete points will depend on the initial point of the particular trajectory as well as on the magnitude of $h$. But as will be shown, the occurrence and stability of the chattering pseudo-sliding mode can be qualitatively described and precise conditions quantitatively determined.

In summary, the 'best' discretisation is used when the switching lines are not crossed, i.e. when $x_{1}(k) s(k) x_{1}(k+1) s(k+1)>0$, and when this condition does not hold, a switching line is crossed and the signed matrix then swapped in accord with (17) and (18). A look-ahead at Figure 4 for example will indicate that the continuous trajectory, stopping at the switching line, is replaced by a discrete trajectory which crosses and recrosses the switching line to give a chattering pseudo-sliding mode. While the discrete points stay correctly on the continuous trajectory before the switching line is reached, the details of the fine structure of the chattering depend on the initial point taken and the magnitude of the stepsize. 


\section{Conditions for pseudo-sliding mode}

We first determine the discrete analogue of the necessary and sufficient conditions (11). A necessary condition for the occurrence of a stable pseudosliding mode is that the trajectories approach the switching line $s=0$ from both sides. There are two possibilities. For a trajectory from region I (see Figure 1) for which $x_{1}$ and $s$ are both positive, the requirement that the trajectory crosses the switching line to region IV implies that in the limiting case, if $s(k)=0$, then the next value evaluated, $s(k+1)$, must be negative. In the same way, from region III for which $x_{1}$ and $s$ are both negative, the next value $s(k+1)$ evaluated after an $s(k)=0$ must be positive. In both evaluations the top sign in (5) must be used. In summary, if $s(k)=0$, and $x_{1}(k)$ and $s(k+1)$ are of opposite sign, then the top sign must be used. In the second case, the requirement that the trajectory crosses the switching line from region IV to I or II to III implies that $s(k+1)$ and $x_{1}(k)$ must be of the same sign when the bottom sign of $(5)$ is used.

The consequence of this argument leads to the formulation of the necessary condition. From (6) it follows that

$$
s(k)=c x_{1}(k)+x_{2}(k)=\left[\begin{array}{cl}
c & 1
\end{array}\right] \mathbf{x}(k),
$$

and hence from (14) that

$$
s(k+1)=[c 1] \exp \left(\mathbf{A}^{ \pm} h\right) \mathbf{x}(k) .
$$

For $s(k)=0$, this yields

$$
s(k+1)=\left[\begin{array}{ll}
c & 1
\end{array}\right] \exp \left(\mathbf{A}^{ \pm} h\right)[1-c]^{T} x_{1}(k) .
$$

With the use of (8), it follows that

$$
s(k+1)=\exp (-\alpha h) \beta^{-1} \sin (\beta h) x_{1}(k)\left(c f-c^{2} \mp a\right) .
$$

The necessary conditions for the existence of a stable pseudo-sliding mode, namely that $x_{1}(k)$ and $s(k+1)$ are of opposite signs for the top sign in (22) and of the same sign for the bottom sign, are simply $c f-c^{2}-a \leq 0$ and $c f-c^{2}+a \geq 0$, equivalent to (13), since $\exp (-\alpha h) \beta^{-1} \sin (\beta h)>0$.

The condition can be made to mimic a discretisation of (12) by recalling that $s(k)=0$, so that $(22)$ can be written

$$
\frac{s(k+1)-s(k)}{\exp (-\alpha h) \beta^{-1} \sin (\beta h)}=x_{1}(k)\left(c f-c^{2} \mp a\right) \text {. }
$$


Since $\exp (-\alpha h) \beta^{-1} \sin (\beta h)=O(h)$, the left hand side of (23) approaches $\dot{s}$ as $h$ approaches zero, so that in the limit of the continuous system the condition (12) is correctly obtained.

A sufficient condition for the existence of a pseudo-sliding mode will be derived as a combination of two conditions. The first is (13) or (23) which guarantees that the asymptote is on the correct side of the switching line (see Figure 2). Although (13) forms by itself a sufficient condition for the continuous case, it does not for the discrete case as a further condition on the magnitude of $h$ is required. This second condition can be determined from the following argument. It is clear that the condition must involve a restriction on the magnitude of $h$, for this controls the sizes of the steps along a trajectory. What is required is that when a trajectory is being stepped out, a step across the switching line must be sufficiently small so that region which forces the return in the direction to the switching line is not over-stepped. This region is bounded by the switching line $s(\mathbf{x})=c x_{1}+x_{2}=0$ and the asymptote

$$
r(\mathbf{x})=(\alpha+\gamma) x_{1}+x_{2}=0,
$$

where

$$
\gamma=(1 / 2) \sqrt{4 a+f^{2}} .
$$

The region is represented in Figure 2 by regions IIB and IVB, and it will be noted that the fifteenth step is approximately from a point on the switching line to a point just out of the region IVB so that the trajectory veers away.

The limiting case occurs when a step is from the switching line to the asymptote, that is, from $s(k)=0$ to $r(k+1)=0$. As in the derivation of (20),

$$
r(k+1)=\left[\begin{array}{ll}
\alpha+\gamma & 1
\end{array}\right] \exp \left(\mathbf{A}^{ \pm} h\right)\left[\begin{array}{ll}
1 & -c
\end{array}\right]^{\top} x_{1}(k),
$$

which leads to

$$
r(k+1)=\left[\left(\mp a+\alpha^{2}+\alpha \gamma-c \gamma\right) \beta^{-1} \sin (\beta h)+(\alpha+\gamma-c) \cos (\beta h)\right] x_{1}(k) .
$$

The condition $r(k+1)=0$ places an upper bound on the value of $h$ given by

$$
H=\beta^{-1} \operatorname{atan}\left[\beta(\alpha+\gamma-c) /\left(a-\alpha^{2}-\alpha \gamma+c \gamma\right)\right] .
$$

Only the top sign is used because $r(k+1)$ is calculated from a previous point in region I or III for a trajectory just straddling region IVB or IIB.

It is interesting to recover the result for the continuous system by taking the limit as $h$ approaches zero. This implies $H=0$ and hence from (28)

$$
\alpha+\gamma-c=0 \text {, }
$$




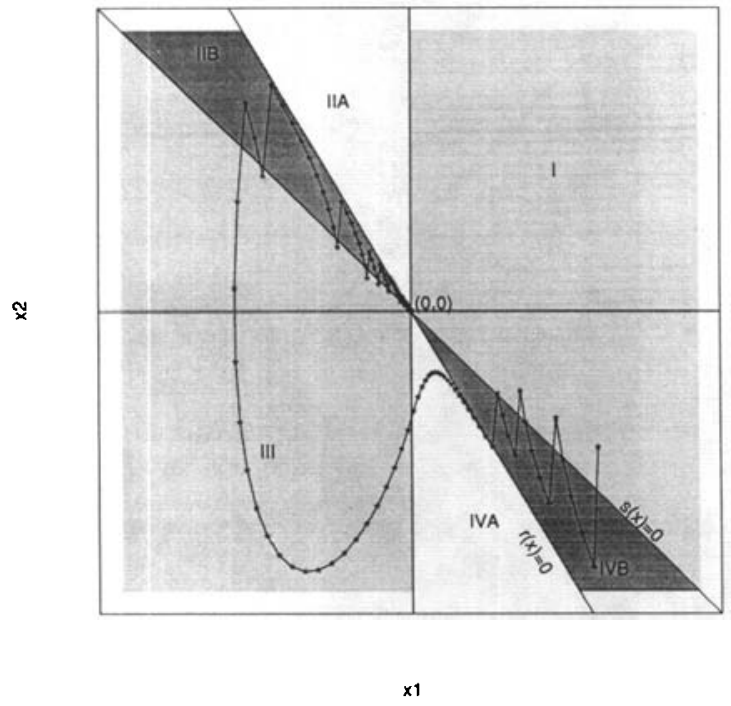

FIGURE 2. The phase plane is further subdivided by the asymptote $r(x)=0$. The heavily shaded regions IIB and IVB correspond to regions where the trajectories move towards the switching line $s(x)=0$. The trajectory shown illustrates a system which is stable but without a pseudo-sliding mode, corresponding to $c=1, a=9, f=-3.49, h=0.049$.

or

$$
c f-c^{2}+a=0 \text {. }
$$

This agrees with the one of the equalities in (13). The other arises from the necessity to maintain a region IVB, which is lost if the line $r(x)=0$ coincides with or lies above the switching line $s(\mathbf{x})=0$.

\section{Numerical results}

The constant $c$ in (2) is positive and the rescaling $x_{2} \rightarrow x_{2} / c, t \rightarrow c t$ enables $c$ to be taken equal to unity for numerical calculations without loss of generality. To explain the scaling procedure, suppose that in the given system $c=2$ in order to give a sliding mode with a time constant of 0.5 seconds and that the system starts from rest with $x_{1}$ representing an angle with initial value 10 degrees. Then in Figure 3 on p. 372, for example, a unit on the $x_{1}$ axis represents $10^{\circ}$, a unit on the $x_{2}$ axis $20^{\circ} / \mathrm{sec}$ and $h=0.002$ a step-size of 0.001 seconds.

Initial values of $x_{1}=1$ and $x_{2}=\dot{x}_{1}=0$ are useful for comparison purposes, representing starts from rest at an initial displacement of magnitude unity. 


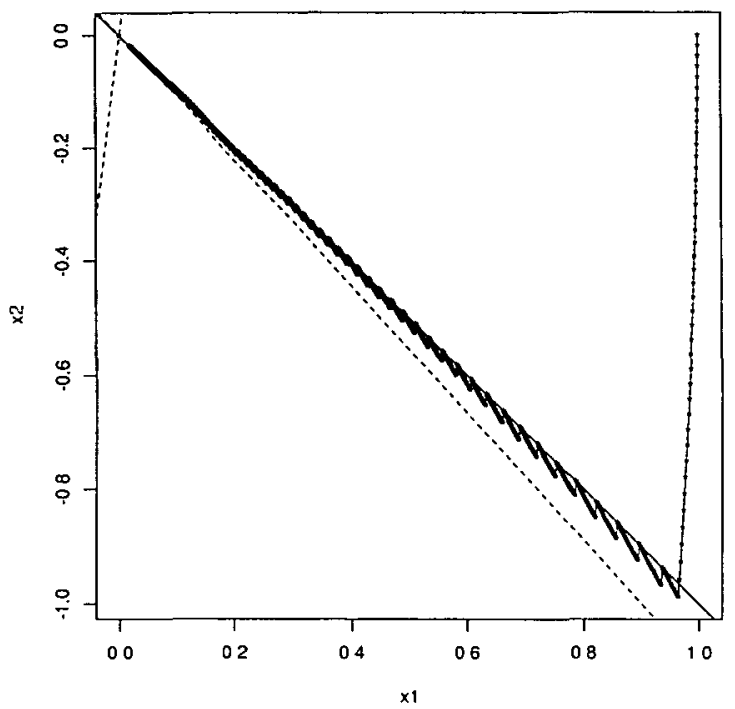

Figure 3. A stable pseudo-sliding mode with the following values: $c=1, a=9, f=-7$, $h=0.002$.

Figure 3 illustrates a stable pseudo-sliding mode with $c=1, a=9$, $f=-7, h=0.002$. The value of $f$ corresponds to 'large' negative friction but is still in the range $-8<f<10$ for a stable sliding-mode for the continuous system (see (13)). The value of the step-size $h$ is less than the upper bound $H \approx 0.00627$ calculated from (28).

Figure 4 illustrates a stable pseudo-sliding mode with the same parameters as in Figure 3 except that the friction is now positive. The 'chattering' tends to be above the switching line rather than below. The value of the upper bound is $H=0.722$, considerably increased.

Figure 5 again illustrates a stable pseudo-sliding mode with the friction changed to $f=-3.49$. The value of the stepsize is taken as $h=0.046$, just less than the upper bound $H=0.0467$. Any overstepping of the region between the switching line and the asymptote is just prevented.

Figure 6 on p. 374 illustrates a stable system without a pseudo-sliding mode. The data is as for Figure 5 except that $h=0.05$, which just exceeds the upper bound. The trajectory appears to be approaching the origin in a pseudo-sliding mode but suddenly veers away to circuit the origin before closing in again. This behaviour is repeated indefinitely. Figure 2 also illustrates a stable system without a pseudo-sliding mode starting from a different initial position, and with $h=0.049$. 


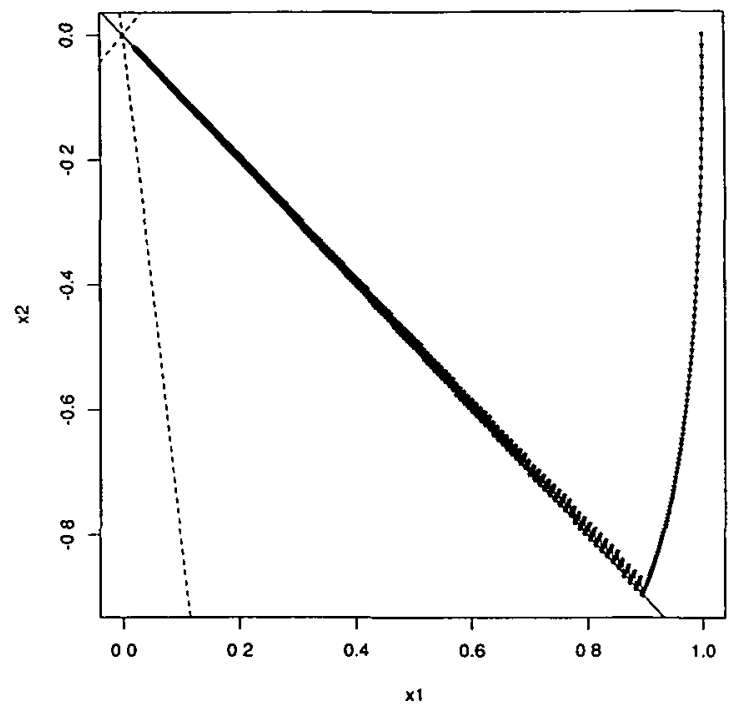

Figure 4. A stable pseudo-sliding mode with the same data as for Figure 3 except that $f=7$, corresponding to positive rather than negative friction.

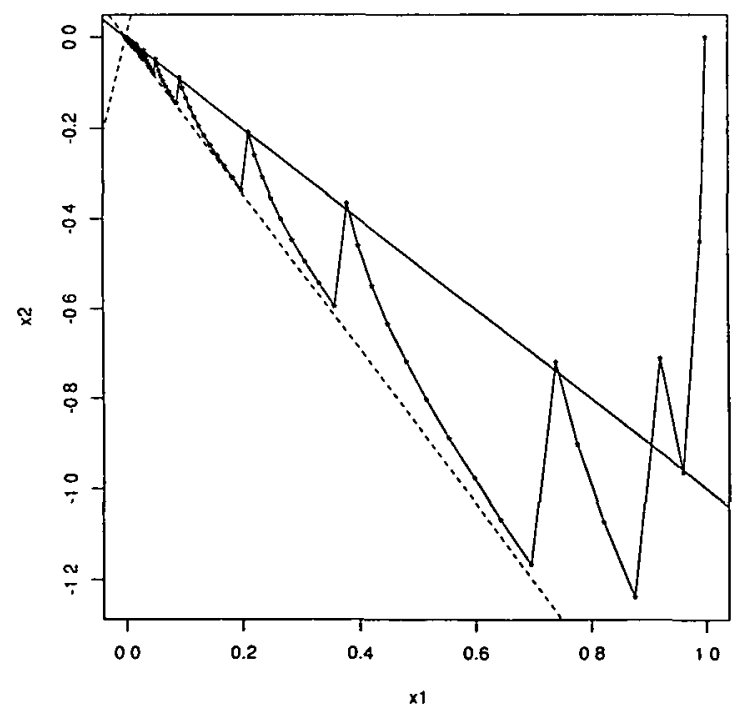

Figure 5. A stable pseudo-sliding mode with the same data as for Figure 2 except that $h=0.046$, just less than the upper bound. 


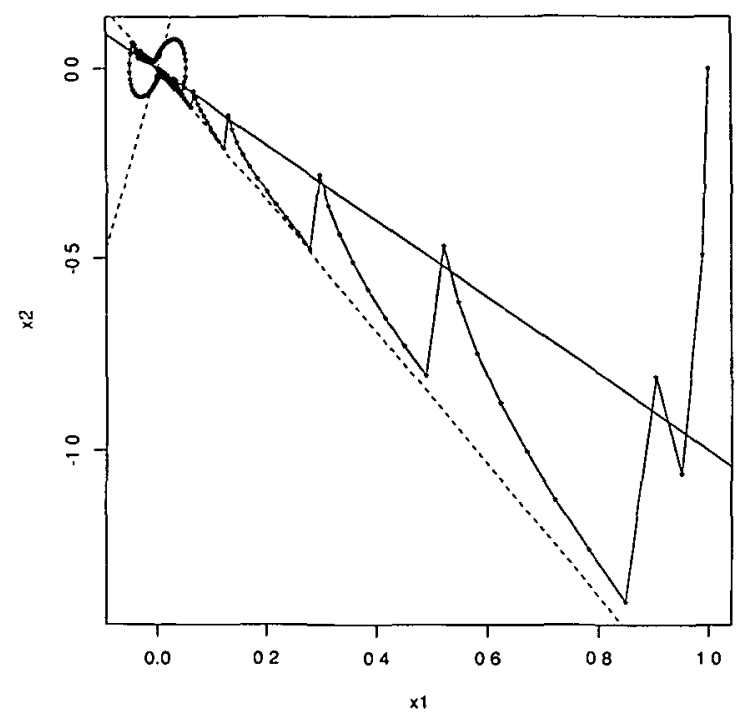

Figure 6. A stable system without a pseudo-sliding mode. The data is the same as for Figure 5 except that $h=0.005$, exceeding the upper bound. Compare with Figure 2.

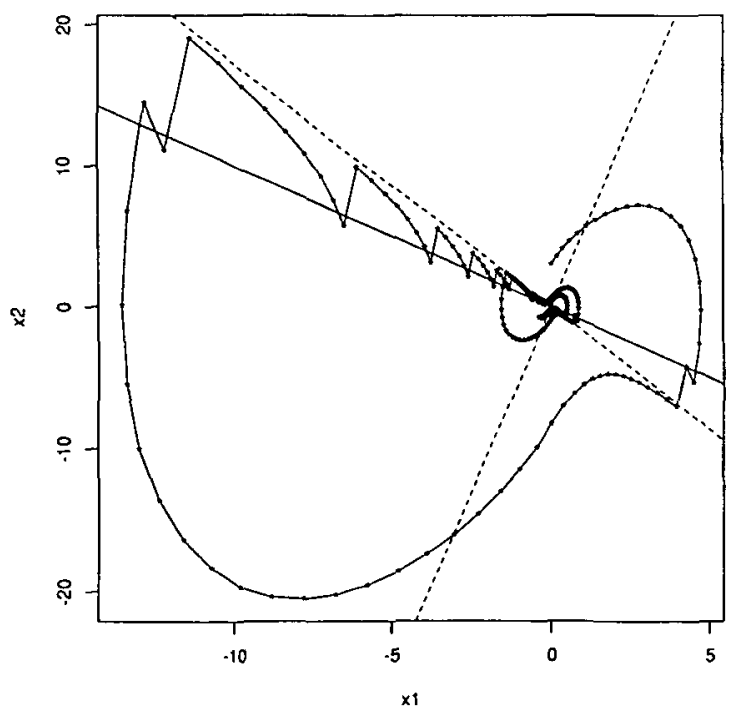

FIGURE 7. A stable system without a pseudo-sliding mode. The data is the same as for Figure 6 except the initial point is taken as $x_{1}=0.01, x_{2}=3.0$. 


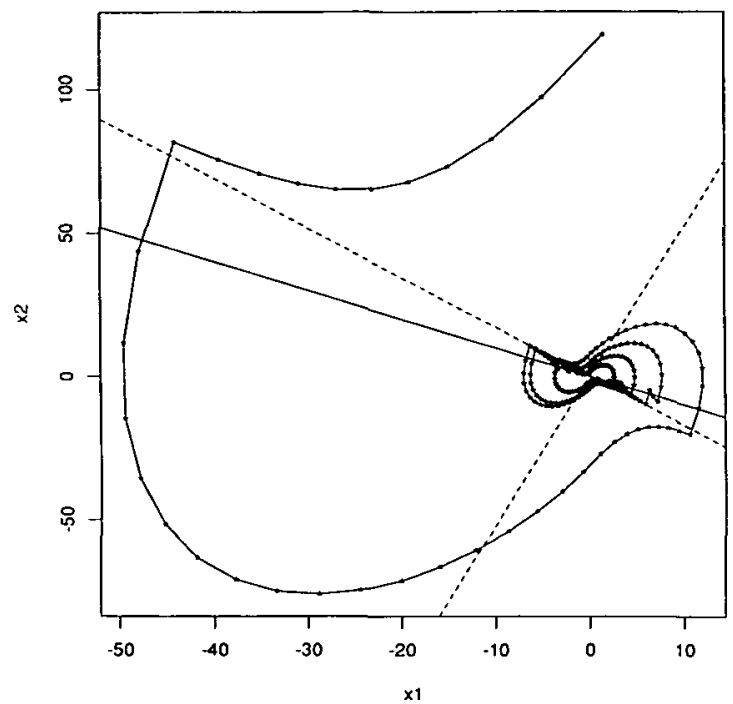

Figure 8. An unstable system. The same data as for Figure 7 except that $h=0.06$, considerably larger than the upper bound.

For Figure 7, the data is exactly the same as for Figure 6 except that the initial point is taken as $x_{1}=0.01, x_{2}=3.0$. The 'small bird in a big bird' picture again corresponds to a stable system without a pseudo-sliding mode.

For Figure 8, the system is unstable, with the same values as for Figure 7 except for $h=0.06$, considerably greater than the bound $H=0.0467$.

\section{Discussion}

The fact that the variable structure system considered in this work is, apart from the switching logic, a linear system with constant coefficients, has enabled a 'best' discretisation to be taken in the sense that from any point not on a switching line the discrete points are taken to be exactly on the continuous trajectory emanating from that point. This does not imply that the solution obtained in a particular case is independent of the initial point or the value of $h$ because changes in these would alter the discrete step the discrete solution takes as it crosses a switching line. But because of the discretisation used a qualitative analysis has proved possible and in particular the exact analysis of the discrete system has enabled necessary and sufficient conditions for the occurrence of a pseudo-sliding mode to be determined. These are 
substantiated by numerical results. The well-known theory of continuous variable structure systems has been confirmed in the limit as the discrete stepsize approaches zero, and Figure 4 for example illustrates how the pseudosliding mode approaches the sliding mode-and gives a more convincing representation than a hand-drawn wiggly line.

Further work is being done on more complicated systems and on applications to the control of robots.

\section{Acknowledgements}

The authors are indebted to the Australian Research Council for an award. One of the authors (R.B.P.) expresses his gratitude to Professor Mike Brady and Dr Ron Daniel for their hospitality at the Robotics Laboratory, Oxford University, where this research was initiated.

\section{References}

[1] E. A. Barbashin, Introduction to the theory of stability (Wolters-Noordhoff, Groningen, 1970).

[2] A. F. Filippov, Differential equations with discontinuous righthand sides (Kluwer, Dordrecht, 1988).

[3] C. Milosavljevic, "General conditions for the existence of a quasisliding mode on the switching hyperplane in discrete variable structure systems", Automat. Remote Contr. 46 (1985) 307-314.

[4] R. B. Potts, "Differential and difference equations", Am. Math. Monthly 89 (1982) 402-407.

[5] V. I. Utkin, "Discontinuous control systems: state of art in the theory and applications" 10th IFAC World Congress on Automatic Control, 1 (Munich 1987).

[6] K-K. D. Young, "Controller design for a manipulator using theory of variable structure systems", IEEE Trans. Syst., Man and Cyb. SMC-8 (1978) 101-109. 